\title{
A practical safety risk model for monitoring program design
}

\author{
J Venter AngloGold Ashanti Australia Ltd, Australia \\ ECF Hamman AngloGold Ashanti Australia Ltd, Australia
}

\begin{abstract} analytically answer questions such as:

- How frequently should slopes be inspected?

- How frequently should prisms be read?

- Should a radar be acquired? If so, which one?

- How many monitoring systems to use? And many more.
\end{abstract}

Many publications are available that provide statements of best practice in terms of open pit slope risk management. However, to date none provide a risk model that demonstrates the risk reduction achieved for applying each of the risk management elements. This leaves the slope stability practitioner unable to

This paper applies the Venter and Hamman (2018a) temporal safety risk model to an open pit in West Africa. The paper demonstrates the use of the model to a small saprolite open pit and concludes with recommendations for slope instability registers to facilitate future back-analysis in terms of this model.

Keywords: safety risk model, monitoring frequency, monitoring system design

\section{Introduction}

Geotechnical engineering for open pit mining relies heavily on monitoring to enable mining companies to safely extract ore without suffering safety or equipment damage. Monitoring allows higher Probabilities of Failure to be safely used as acceptance criteria but when combined with ongoing data collection, is the only protection available against unknowns. Monitoring is the last line of defence against slope instability, and its relative importance increases as other forms of defence against geotechnical risk such as face mapping, in-pit drilling, structural mapping, etc. and the subsequent data interpretation are exhausted.

A significant problem that faces the open pit geotechnical practitioner is to know how much monitoring is required to meet a given safety risk acceptance criteria. Contained in this problem are questions such as:

- How does prism monitoring contribute to personnel safety?

- Is a slope radar required?

- Is only a slope radar sufficient?

- How often should someone check the slope radar or prism monitoring data?

- How important is the evacuation plan?

- What benefit is there in a waiting period following blasting or rainfall before mining again?

- How important is correct mechanism identification to slope monitoring?

- How frequently should supervisors conduct inspections? Among many others.

To answer these questions, Venter and Hamman (2018a) proposed a temporal safety risk model that is based on a timeline of events leading up to slope instability. The power of the Venter and Hamman (2018a) model is that the inputs are simple to estimate or calculate using back-analysis of past failures. This gives it a distinct 
advantage over models that require probabilities as inputs. As a result, the need for workshops are eliminated and the need for subjective inputs eliminated or at least greatly reduced and simplified.

There were, however, two limitations with the model proposed by Venter and Hamman (2018a) that are addressed in this paper.

The first limitation is that the graphic output available from the model proposed by Venter and Hamman (2018a) was not available, and that made it difficult to interpret and understand the results obtained. After using this tool at several mine sites, a meaningful graphical output was developed that shows the timeline of events underpinning the model. The graphical output is presented in this paper.

The second limitation is that the Venter and Hamman (2018a) paper only applied the model to a fictitious case study and it would have been more interesting to see it applied to a real case study. This paper addresses that limitation by demonstrating the model with a small saprolite open pit at an AngloGold Ashanti operation in West Africa. While the example pit is a small saprolite pit with limited monitoring, it represents only one of the many types of open pit monitoring problems that can be encountered, so limitation two is only partially addressed.

In spite of only partially addressing the case study limitation, several important lessons were learned with regard to how the model could be applied and the type of information that needs to be collected for slope instability registers. These lessons are presented in this paper.

The proposed model, along with the new graphic output, are presented in the following sections, followed by the example case study. Finally the lessons learned are discussed and presented in the form of suggested slope instability register inputs for future use.

\section{Proposed model}

Venter and Hamman (2018b) proposed that one could view geotechnical risk management as consisting of four risk management gates or stages. Each risk needs to pass through all stages before a safety consequence or equipment damage consequence is reached. The four stages are design, validation, creep and collapse. Figure 1 shows all four stages at the top and then expands the internal workings of the collapse stage which is the topic of this paper.

In summary, the four stages of a geotechnical hazard are:

1. Design: The design stage represents the time period before mining commences. The actual open pit risk during this period is zero (even though a report may exist presenting a Probability of Failure (PF)) as there is no excavation yet. If this design is for a cutback, any risk associated with the existing excavation still belongs to the previous design and not the cutback design. The major features of the design period are that:

a. There are practically no limitations imposed on the potential design except those imposed by the project owner.

b. The information available for a design is often limited to boreholes and surface mapping as no excavation may be present. Even if existing pits are nearby, such as during a cutback, details of the structural variations at the exact location of the slopes are likely to be incomplete.

2. Validation: The validation stage represents a period of time following the blasting of the first bench during which the prism and other low-intensity monitoring tools can be installed, and measurement histories started, and during which early information from in-pit mapping and mining experience can be used to validate the design and identify previously unidentified hazards. As this period ends for a slope once the first significant instability is initiated, it represents a golden period of proactively detecting potential instabilities with sufficient time to change pit designs while maintaining schedule flexibility. The main purpose of slope monitoring in this stage should be to detect the onset of the creep stage. 
3. Creep: The creep stage represents the period of time after initiation of a significant slope instability, but before it is too late to prevent the instability through slope design changes or remediation measures. The exact start of the creep stage may not be precisely pinned down due to lack of information or imprecise equipment. The exact end of the creep stage is as much a function of the mine's ability and willingness to intervene as it is of the slope's mechanical disposition to collapse. For example, the life of the creep stage can be shortened by deciding not to build a buttress even if it technically would have worked. The end of the creep stage represents the end of all opportunities to limit economic damage by changing the slope geometry. The purpose of slope monitoring during the creep stage is to detect the onset of the collapse stage and to provide inputs for the safety risk model presented in this paper.

4. Collapse: The collapse stage represents the final stage before collapse and is entered into the moment that slope collapse becomes inevitable. At this point, economic damage becomes inevitable and is locked in. The only control remaining is to continue mining as long as mine management is comfortable with the level of safety risk, based on the quality of real-time monitoring available.

Venter and Hamman (2018a) proposed their initial model only for use to determine critical monitoring requirements during the collapse stage in the life of a slope instability. However, subsequent use demonstrated that the Venter and Hamman (2018a) model can be used during all stages of slope stability. This paper demonstrates that through the practical example.

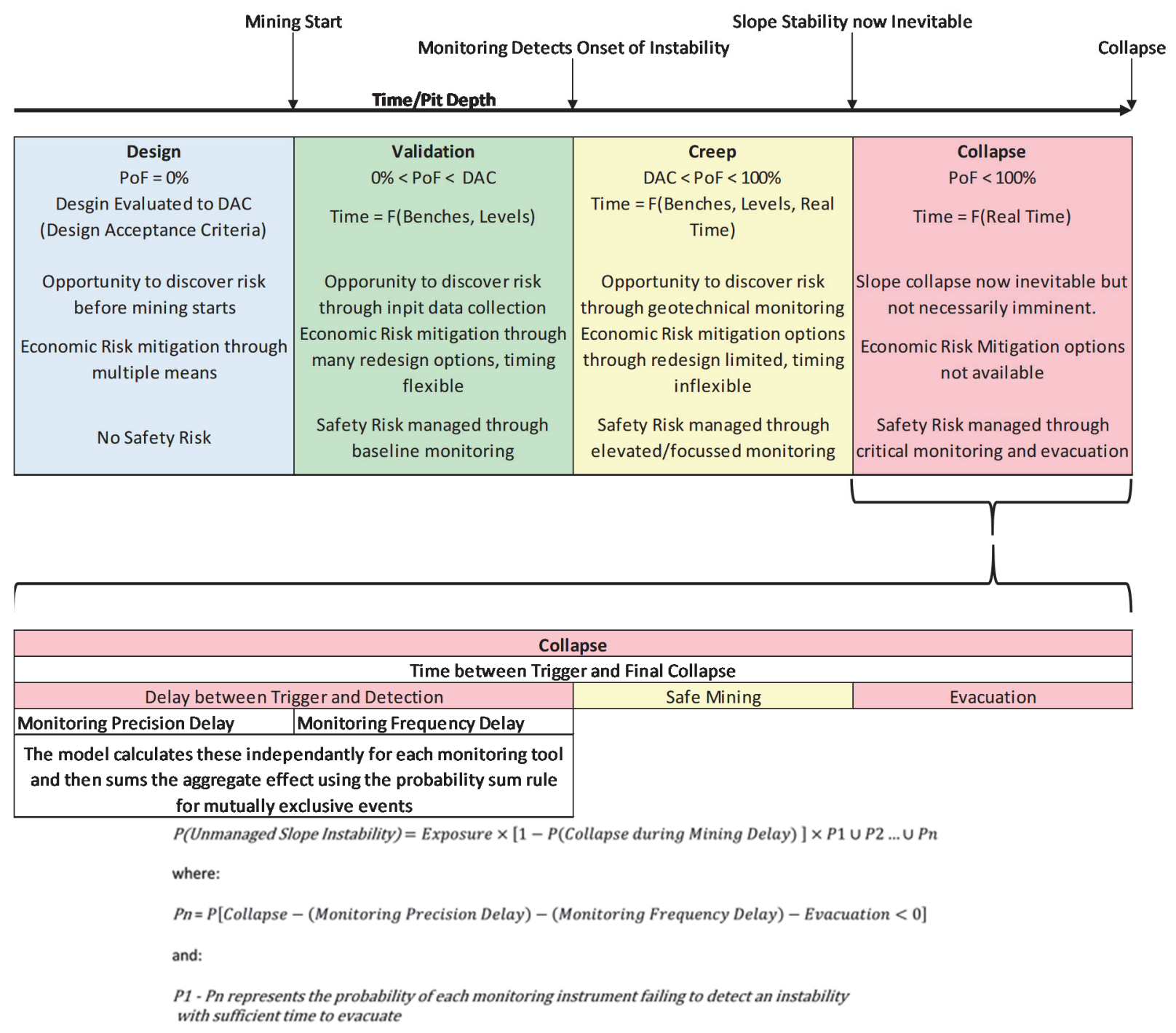

Figure 1 Proposed risk model 


\subsection{Model concept}

The underlying mechanics of the Venter and Hamman (2018a) model relies on time as a basic unit of measurement. The basic reasoning is that there is a certain amount of time remaining between the triggering moment and a potential slope instability. An assessment of the remaining time to slope instability and corresponding PF can be made with some confidence either through experience, or through direct measurement of similar slopes elsewhere with consideration of the stages presented in Section 1 . The model assumes that slope instability is detected and managed using slope monitoring through a five-step process starting with the present and leading up to slope instability. A duration for each step is required as model input. The durations for each step are summed in the model and compared to the estimate of the remaining time to failure. If there is less time remaining than it takes to go through these steps, the slope instability is deemed an 'unmanaged slope instability'. As all the inputs barring PF and exposure are provided as distributions of possible values, this calculation is repeated as a Monte Carlo analysis for a thousand realisations. The percentage of realisations where the instability management process takes longer than the remaining time to slope instability is considered the probability of an unmanaged slope failure.

\subsection{Model inputs}

Each of the durations of the five steps are entered as a minimum, best estimate and maximum duration. The model then uses the beta distribution in the Monte Carlo simulation to produce the outputs. The five steps used in the model with corresponding inputs are as follows:

- Time delay before entering pit following expected trigger: In certain cases, the collapse trigger may be associated with blasting or rainfall; both typical events where the pit would have been evacuated as standard practice. This is to capture the time that it takes from the trigger event to when the people and equipment are back in the pit in the danger zone. Increasing this delay will reduce the risk as the likelihood of trigger induced collapse is greater immediately after the trigger and reduces with time.

- Delay from trigger to final collapse: This represents the full period of time available from triggering a slope stability to final collapse. The trigger in this case is the real trigger, not detection of the trigger. It is envisaged that an estimate of this time period can be made through looking at case histories of similar slopes at the same mine or in the same region, or from numerical modelling.

- Delay between trigger and detection: This is a measure of monitoring precision, related to the capacity of the equipment and procedures, and expressed as typical consecutive time intervals required to identify and detect the collapse trend. The user enters the minimum, maximum and estimated time that each monitoring method would require to detect the trend (e.g. a shift boss or supervisor doing an inspection each 12 hour shift would typically notice something after two inspections). Therefore the estimated value would be 24 hours. The minimum can only be 12 hours and the maximum can be taken as 48 hours. Alternatively, prisms being read to $10 \mathrm{~mm}$ precision will take five days after starting to accelerate at $1 \mathrm{~mm} /$ day before the prisms will detect the change in trend. This time represents a delay caused by monitoring imprecision. The current model makes provision for entering six different monitoring methods.

- Monitoring interval: This describes the monitoring frequency for each method as opposed to the precision. To use the previous prism example, if the prisms were read daily and a possible trend change could only be detected after five days (if measured continuously), the monitoring frequency assumes that the five days expire immediately after a reading and will only be detected a full reading later. So for this example, for daily readings, the trend change will be detected after six days. Again, the model makes provision for entering the monitoring interval for six different monitoring methods. 
- Evacuation: This is the actual duration of an evacuation from the moment the alarm is raised to the moment the last personnel and equipment leaves the danger zone. This is entered as a fixed value, and this value should be the target for practice runs in the pit, or a reflection of actual exercises.

The model does the following calculations that can be used for decision-making and understanding.

- Personnel/equipment exposed: The personnel exposed calculation is simple and based on the percentage of time in a day that personnel is exposed. The equation is [(delay from trigger to final collapse) - (time delay before entering pit following trigger)] $\times$ (daily personnel exposure).

- Likelihood of warning provided: This is a measure of monitoring system efficiency only but excludes evacuation time (i.e. a successful score here implies early warning would be provided but not necessarily with sufficient time to evacuate). The calculation for a single monitoring instrument is $\mathrm{P}[($ delay from trigger to final collapse) - (delay between trigger and detection) - (monitoring interval) > 0] based on a Monte Carlo simulation. The total system probability is simply the union of this result for all employed monitoring systems.

- Evacuation in time: This is a measure of whether sufficient warning is provided to evacuate in time. The calculation is P[(delay from trigger to final collapse) - (delay between trigger and detection) (monitoring interval) - (evacuation time) $>0$ ] based on a Monte Carlo simulation.

- P(unmanaged slope movement): This is the final result and is the union of the evacuation in time values for all monitoring instruments. The union was used as only one instrument needs to be correct for the alarm to be triggered.

\subsection{Graphical model output}

The graphical model output and an example of which is shown as Figure 2, which shows the timeline of events from the triggering moment to potential slope instability as probability density on the vertical access and time in hours on the horizontal axis. The different lines on the graph are as follows:

- Solid red line: This represents the distribution of likely time to slope instability from the triggering moment.

- Dotted purple box: This represents the 'no exposure window' which is the exclusion period the mine uses following rain, blasting or other triggers.

- Fine dotted lines: These represent the estimates of when each of the monitoring tools is likely to show signs of impending slope instability.

- Solid lines: These represent the time after the triggering moment when each instrument would have been read and the signs of impending slope instability detected. This will be equal to the fine dotted lines plus the measurement interval.

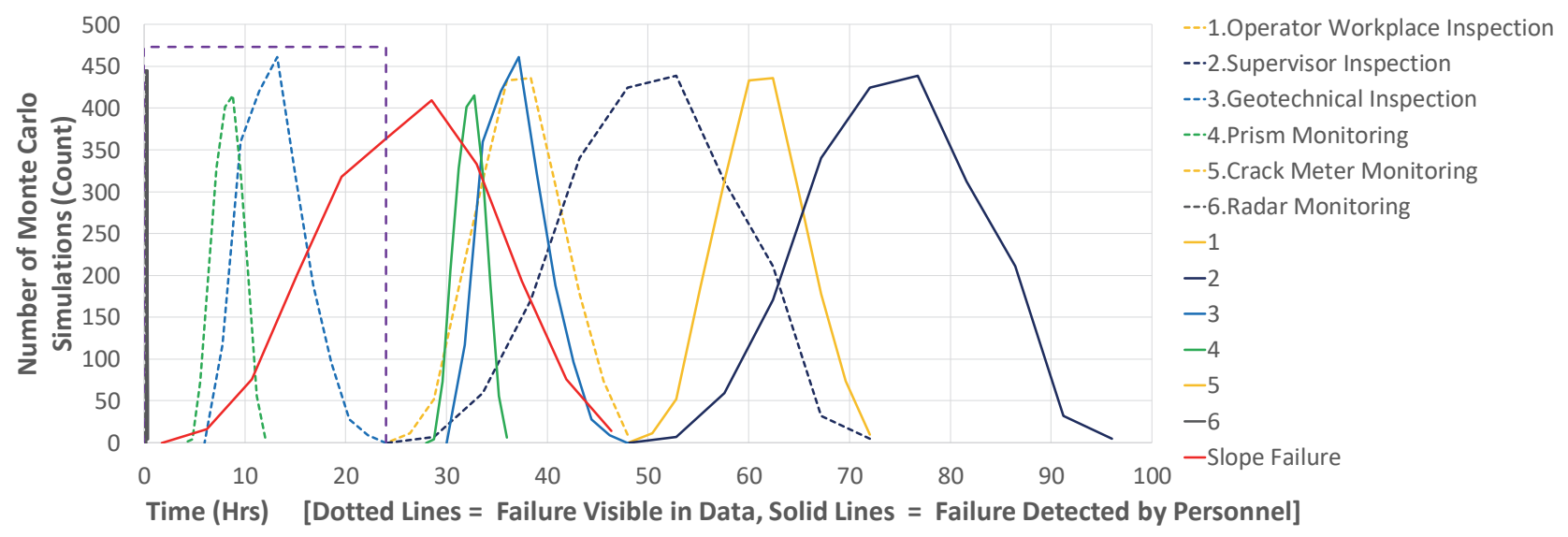

Figure 2 Example graphical output 


\section{Example application}

To demonstrate how the model works, it was applied to the example open pit in West Africa. The selected pit is an ideal small example as it was mined in a short period of time (11 weeks) and experienced two overall scale slope instabilities while mining the last bench driven by an influx of groundwater. These instabilities were well-managed by site and an evacuation occurred days before final collapse. An actual triangulation, drone image, and photo of the instabilities are presented as Figures 3 and 4.

Due to the short duration, such small pits do not provide sufficient time to develop a comprehensive prism monitoring system and the small size is insufficient to justify acquiring a slope radar. The example pit therefore presents a good opportunity to test the model and see whether a reasonable monitoring program could be designed that meets international open pit safety criteria while maintaining a level of effort commensurate with the small and remote nature of the pit.

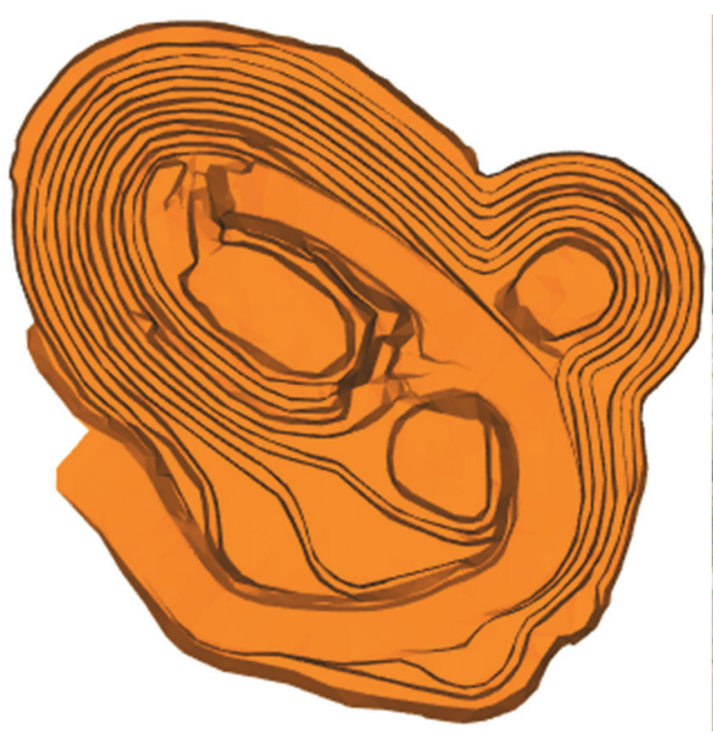

(a)

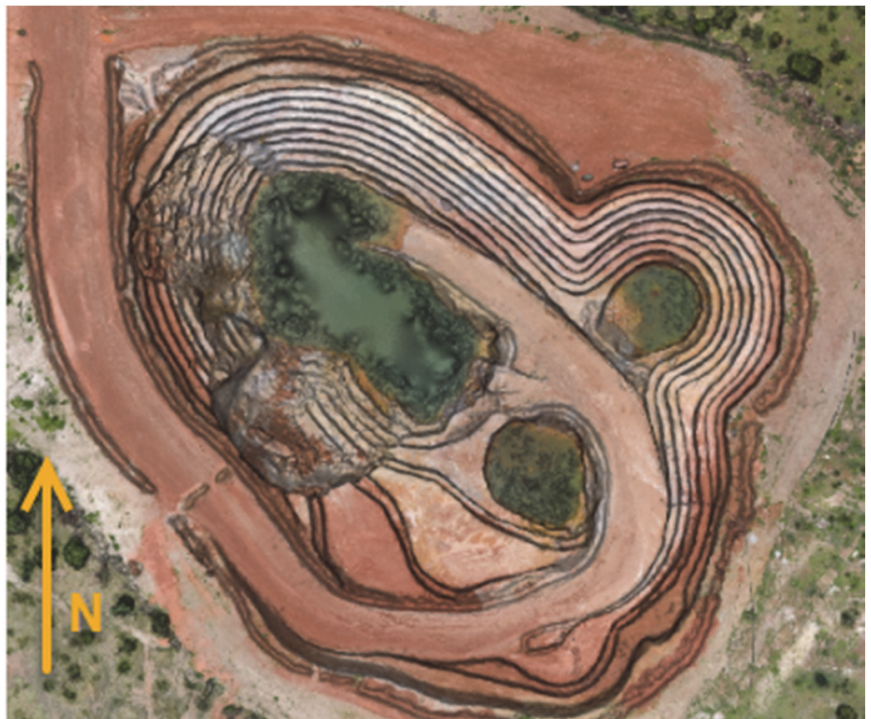

(b)

Figure 3 (a) Actual triangulation; (b) Drone photo

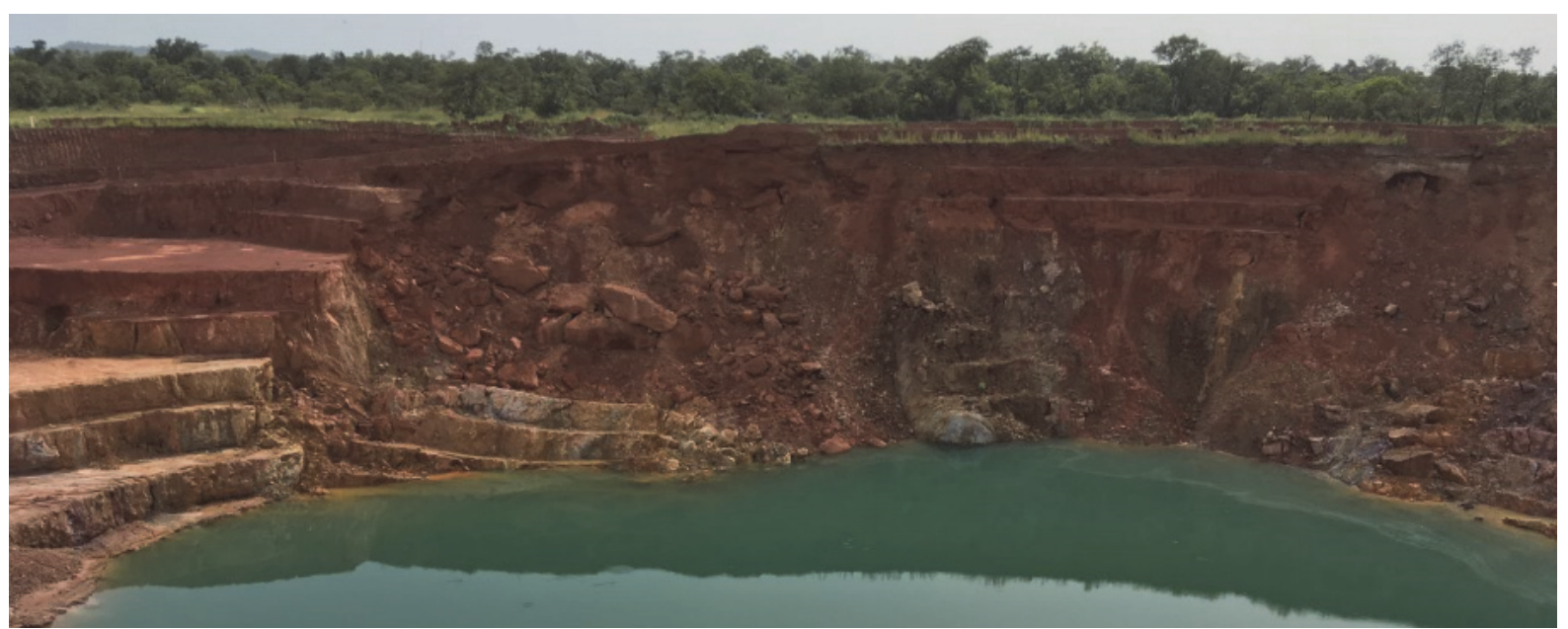

Figure 4 Slope instability photo

Three scenarios were analysed for the example pit as could be expected while mining a small pit: shallow operating conditions ( $\mathrm{PF}=<1 \%$ ), deep operating conditions (PF $=20 \%$ ) and certain slope collapse conditions $(\mathrm{PF}=100 \%)$. The assumptions for each of these scenarios are now presented. 
The following assumptions were used in the safety risk model:

- $\mathrm{PF}=<1 \%$ for shallow operating conditions, $20 \%$ for deep operating conditions and $100 \%$ for certain slope collapse conditions.

- General personnel exposure $=50 \%$ (one $12 \mathrm{hr}$ day shift).

- Time delay before entering pit following potential trigger $=24 \mathrm{hrs}$ after rain. As the example pit is a saprolite pit, no blasting is used.

- Delay from trigger to final collapse.

- Shallow operating conditions: Three days, six days and nine days respectively for the minimum, expected and maximum delay from trigger to collapse.

- Deep operating conditions: Three days, six days and nine days respectively for the minimum, expected and maximum delay from trigger to collapse. The two normal operating condition scenarios have the same values here as, in both cases, the slope has not yet reached the creep stage and will have to pass through the full creep and collapse stages.

- Certain slope collapse conditions: Assuming that the collapse phase is shorter than the creep stage, the delays for this stage were taken as zero days, one day and two days. This is similar to the period between detection and collapse for the example pit.

- Evacuation duration = 12 minutes due to the small pit size and short distance line-of-sight communication possible.

- Monitoring program.

- Shallow operating conditions: Daily supervisor inspection at the start of shift.

- Deep operating conditions: Twice daily supervisor inspection at the start of shift and again at the end of shift.

- Certain slope collapse conditions: 24 hour stand-off after detection of cracks, daily supervisor inspection, daily operator inspection, daily geotechnical inspection, daily prism monitoring and a radar scan every six minutes (details are provided as Table 1). Naturally, this scenario is not practical for such a small pit.

Table 1 Monitoring tool inputs for the certain slope collapse condition scenario

\begin{tabular}{|c|c|c|c|c|}
\hline \multirow[t]{2}{*}{ Monitoring tool } & \multicolumn{3}{|c|}{ Delay between trigger and detection (hrs) } & \multirow{2}{*}{$\begin{array}{l}\text { Monitoring } \\
\text { interval (hrs) }\end{array}$} \\
\hline & Minimum & Expected value & Maximum & \\
\hline $\begin{array}{l}\text { Operator workplace } \\
\text { inspection }\end{array}$ & 12 & 24 & 36 & 24 \\
\hline $\begin{array}{l}\text { Supervisor } \\
\text { inspection }\end{array}$ & 12 & 24 & 36 & 24 \\
\hline $\begin{array}{l}\text { Geotechnical } \\
\text { inspection }\end{array}$ & 2 & 8 & 12 & 24 \\
\hline Prism monitoring & 1 & 3 & 6 & 24 \\
\hline Radar monitoring & 0.1 & 0.2 & 0.3 & 0.1 \\
\hline
\end{tabular}


The results of the analysis are presented as Figures 5 to 7 .

A design approach is required for determining acceptable risk. The mining industry strives for zero harm and no fatalities. However, it is a reality that such an eventuality may occur. As a consequence, for design purposes, the annual probability of a fatality is one guideline adopted in a number of disciplines such as dam safety, motor vehicle accidents, home accidents, etc. The safety standards proposed by Wesseloo and Read (2009) are presented as Figure 8 with the results superimposed.

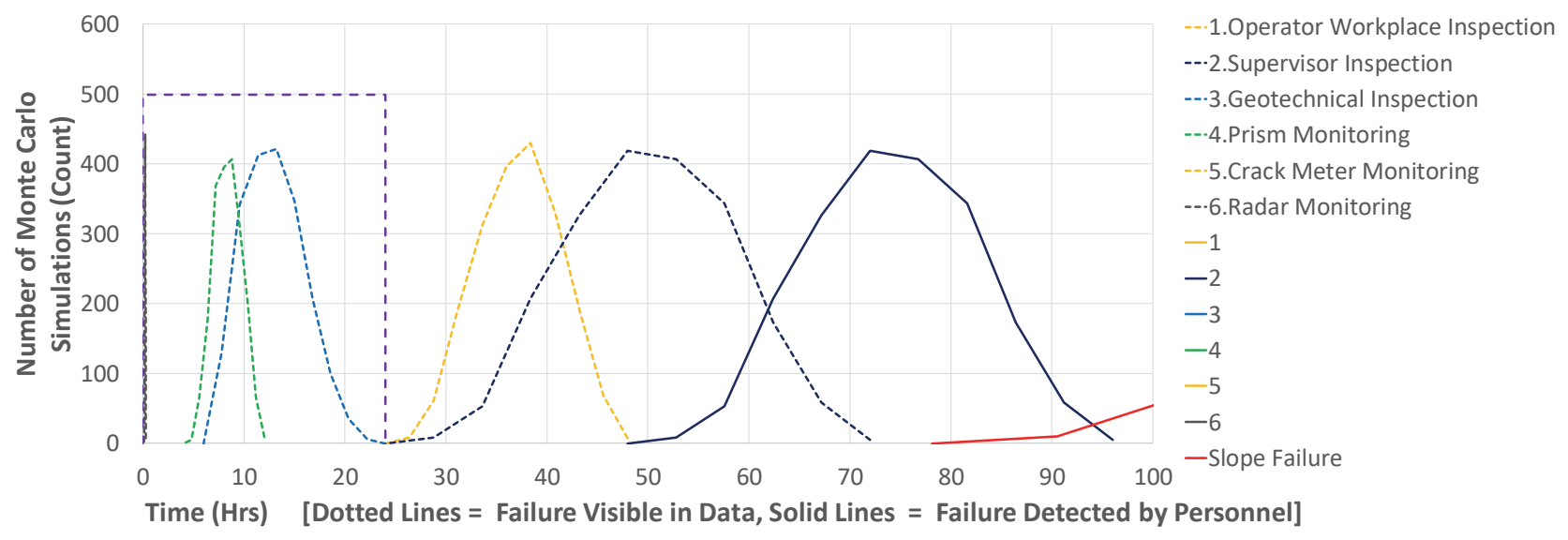

Figure 5 Shallow operating conditions output

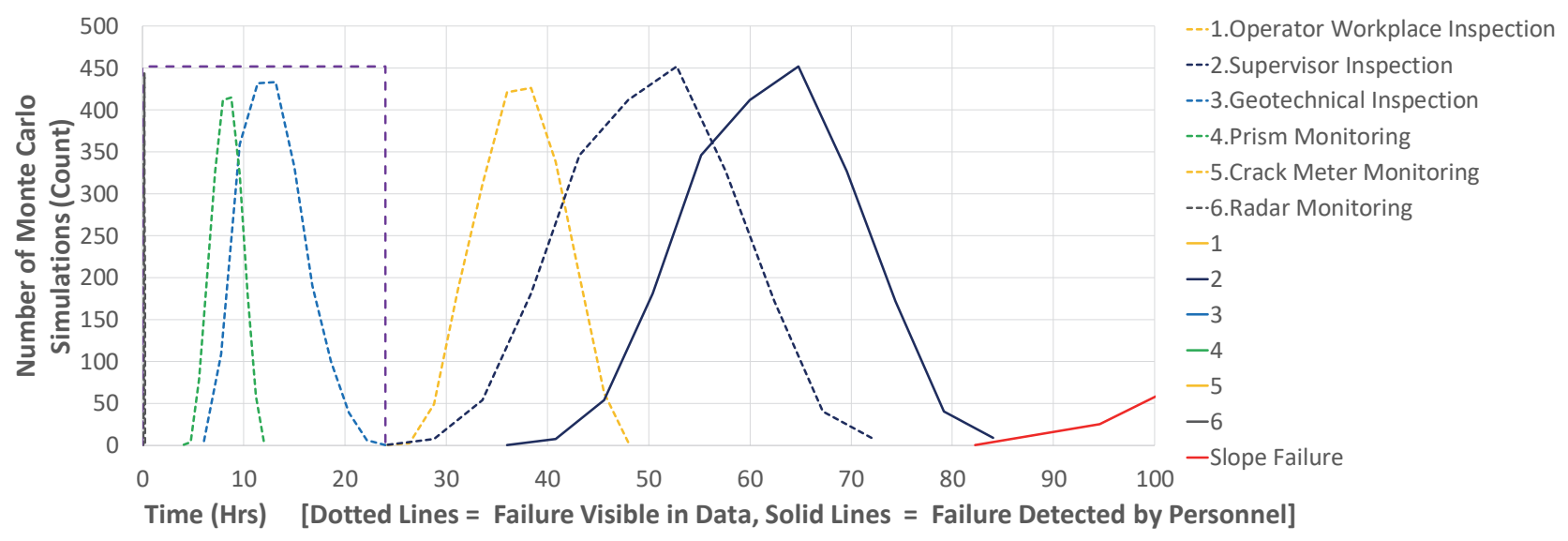

Figure 6 Deep operating conditions output

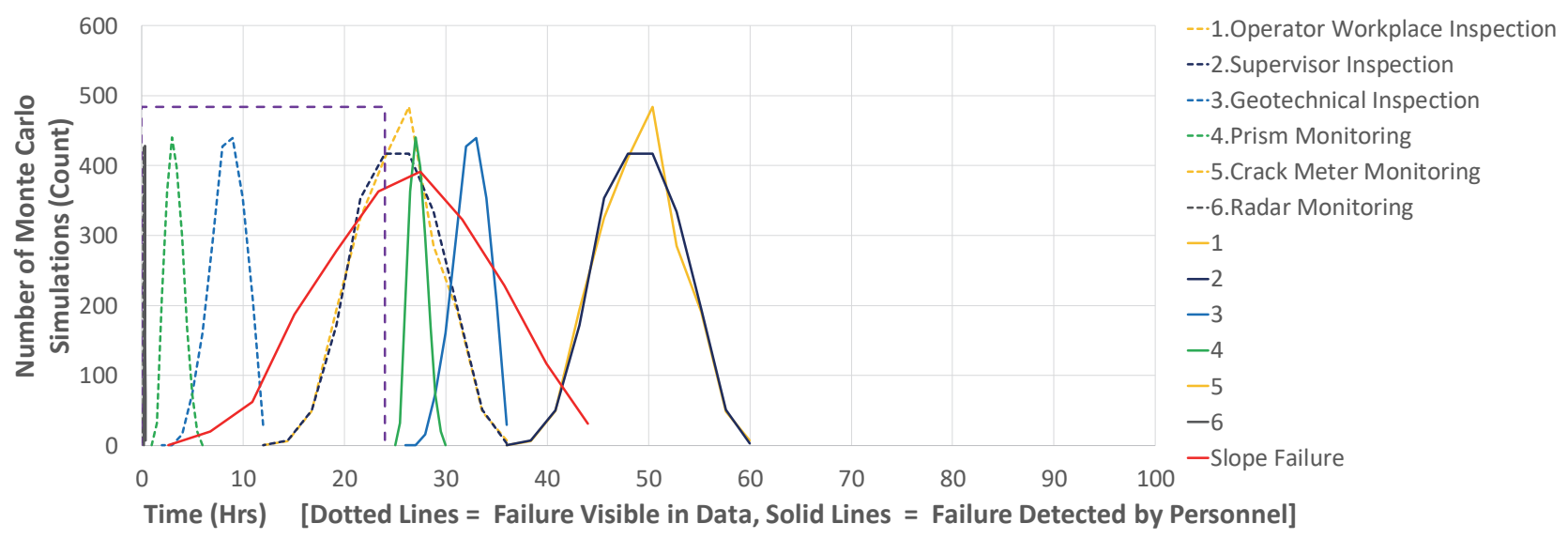

Figure 7 Certain slope collapse operating conditions output 


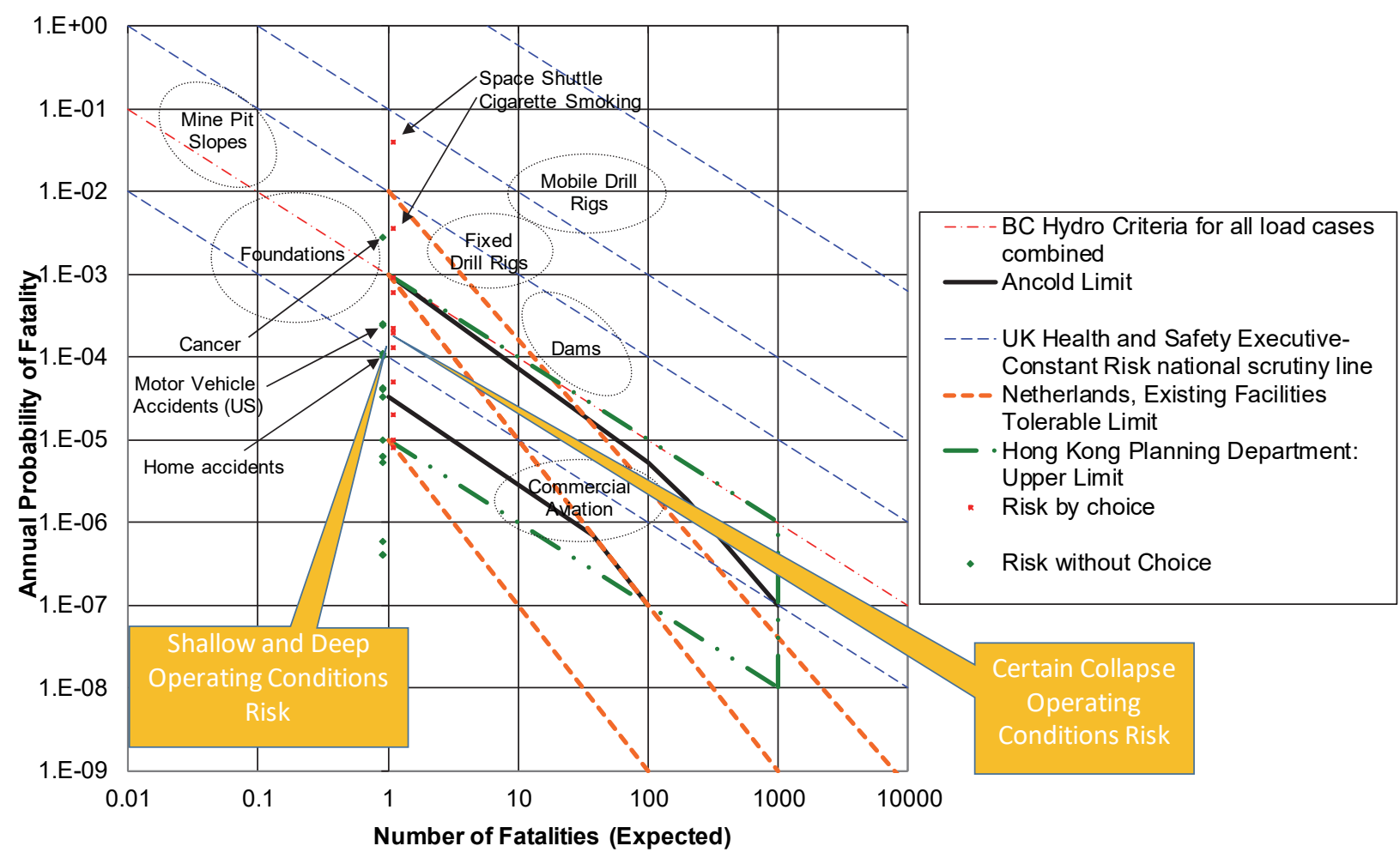

Figure 8 Results superimposed onto safety standard

The $\mathrm{P}$ (unmanaged slope instability) for the shallow and deep operating scenarios are $1 \times 10^{-4}$, while the $\mathrm{P}$ (unmanaged slope instability) for the certain slope collapse scenario is $3 \times 10^{-4}$. These risk values have been superimposed on Figure 8 for comparison purposes. Comparing these risk values to the Wesseloo and Read (2009) guideline is somewhat conservative as Wesseloo and Read (2009) is framed in terms of expected fatalities per annum, while the calculations in this paper are framed in terms of unmanaged slope instability. The conservatism is introduced in that not all unmanaged slope instabilities result in fatalities. The assumption of possible fatalities is, however, maintained.

The results in Figure 8 show that the two normal operating scenarios meet safety standards with a daily supervisor inspection for shallow mining and two supervisor inspections for deep mining below the water table. The certain slope collapse scenario fails to meet safety standards even with a full monitoring program commensurate with much larger pits. The reason for this result is the short expected failure duration used (Figure 7). More precise failure duration data may have resulted in a reduced risk profile. However, this data was not available, and is commonly not available for many small pits.

\section{Discussion}

This paper set out to apply the Venter and Hamman (2018a) temporal risk model to the example open pit in West Africa and to demonstrate the graphical output that has been added since the publication of Venter and Hamman (2018a). In addition, the paper showed that small saprolite pits can be managed according to international safety standards such as those presented by Wesseloo and Read (2008a) by using only inspections by partially trained personnel. Such pits can, however, not be mined safely after cracks have been detected due to the short possible duration of slope instabilities developing. This last point however may change if more slope instability data was available. The monitoring frequencies compare favourably with those proposed by Call (1992) who suggested daily inspections and monthly crack maps and prism monitoring for both the shallow and deep mining scenarios. Naturally, given the 11 week duration of mining, monthly prism monitoring has no value and can be dispensed with along with the monthly crack maps. For the certain slope instability scenario, Call (1992) proposed daily inspections, weekly crack maps and weekly prism 
monitoring. This is much less stringent than proposed by the Venter and Hamman (2018a) model but would have only achieved the as low as reasonably possible (ALARP) region in Figure 8. Such little effort for such high risk may have been acceptable in 1992, but may no longer be as much better technologies have since become available.

\section{Conclusion}

This tool forms part of a larger risk model, of which the sole purpose is to maximise the economic gain while managing the safety impact to acceptable levels. The activities in the economic risk model deal with all states up to the trigger point of collapse. It should be noted that, ultimately, the operations should be operating within the bounds of the economic risk model. However, that is not always achievable, hence this is when the safety risk model comes into play. This tool provides the practitioner the means to demonstrate the effectiveness and efficiency of the monitoring methods employed on the mine.

Applying the Venter and Hamman (2018a) temporal safety risk model to the example open pit also showed the value of collecting more information about slope instabilities than is commonly the practice. For this reason, it is proposed that the following items be added to slope instability registers as a bare minimum:

- Slope instability name.

- Date of instability initiation (crossing from validation to creep stage).

- Significant dates such as stick slip events.

- Date of trigger event such as blasting or rainfall.

- Date of collapse phase initiation.

- Date of instability.

- Rainfall before instability.

- Volume of instability.

- Slope height at time of instability.

- Slope angle just before instability.

- Runout distance of failed material.

- Failure mechanism instability.

Finally, this paper demonstrated the use of the Venter and Hamman (2018a) model by applying it to a small saprolite pit with modest monitoring effort. It would, however, be interesting to also apply it to more elaborate pit slopes with much more rigorous geotechnical monitoring.

\section{References}

Call, R 1992, 'Slope stability', in HL Hartman (ed.), SME Mining Engineering Handbook, Society for Mining, Metallurgy \& Exploration, Englewood, pp. 881-896.

Venter, J \& Hamman, ECF 2018a, 'A temporal safety risk model for open pit slope management', Proceedings of Slope Stability 2018, Asociacion Nacional de Ingenieros de Minas and Colegio Oficial de Ingenieros de Minas del Sur, Seville.

Venter, J \& Hamman, ECF 2018b, 'Determining the optimum slope angle using an integrated mine planning approach', Proceedings of Slope Stability 2018, Asociacion Nacional de Ingenieros de Minas and Colegio Oficial de Ingenieros de Minas del Sur, Seville.

Wesseloo, J \& Read, J 2009, 'Acceptance criteria', in J Read \& P Stacey (eds), Guidelines for Open Pit Slope Design, CSIRO Publishing Collingwood, pp. 221-236. 